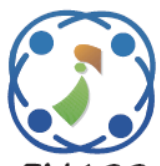

\title{
Promising Gains of 5G Networks with Enhancing Energy Efficiency Using Improved Linear Precoding Schemes
}

\author{
Dheyaa Jasim Kadhim ${ }^{1 *}$ \\ ${ }^{I}$ Electrical Engineering Department, University of Baghdad, Iraq \\ * Corresponding author’s Email: dheyaa@ ceong.uobaghdad.edu.iq
}

\begin{abstract}
Massive multiple-input-multiple-output (MIMO) technology offers high spectral efficiency (SE) using simple schemes while it is also especially expected to offer high energy efficiency (EE). Then to capture promising gains expected from the $5 \mathrm{G}$ networks, this work proposes two improved versions of famous linear precoding schemes, i.e., MRT (maximal-ratio-transmission) and ZF (zero-forcing). To enhance and evaluate the energy efficiency performance in massive multi-user mimo (MU-MIMO) system, we considered here imperfection base station (BS) channel state information. Additionally, our proposed improved versions of linear precoding schemes are taking into account power circuit consumption and transmit power. Simulation results have shown that the improved ZF precoding scheme achieves higher EE as compared to improved MRT precoding scheme, while the results showed that improved MRT precoding outperforms ZF precoding within small BS number of M-antenna deployment comparatively to number of $\mathrm{K}$ terminals (from 20 to approximately 40 antennas). Previous results had been further shown that without circuit power consideration, the energy-efficiency was increasing without limit. In contrast to this belief in the literature, our model shows that energy efficiency is not unbounded when BS antennas increase very-large. We found that around 100 active antennas serving tenth of autonomous users simultaneously showing that energy-efficiency cannot be optimal at low signal to noise ratio (SNR) region, but in region where an appropriate interference-canceling processing, ZF precoding is extremely suitable over an interference-limited processing, MRT precoding. Finally, the results demonstrated and confirmed that an efficient-energy can be achieved by allowing so more and more users to access the channel simultaneously, thanks to massive MIMO that illustrates $5 \mathrm{G}$ promising gain expectations.
\end{abstract}

Keywords: Massive MU-MIMO, Energy efficiency, Achievable rate, MRT precoding, ZF precoding.

\section{Introduction}

In mobile communication system, if the base station can communicate with many users at the same time, such system is called multi-user multiple input multiple output (MU-MIMO) [1]. In such system, the base station (BS) and end users may equip with more small antennas on transceivers to obtain better performance. MU-MIMO system used the principle of space division multiple access (SDMA) to send simultaneously multiple parallel connections in the same time and frequency resource [2].

MU-MIMO system has some important advantages for cellular networks such as: (1) Increasing data rate by sending individual data streams to several users at the same time, which is referred as multiplexing gain; (2) Guarantee connection availability, since the existence of more antennas can provide so many communicating links so the radio signals may spread through, which is referred as diversity gain; (3) Enhancement of energy efficiency, since BS has the ability to direct the transmitted power spatially and exactly where each terminal is incident, the aggregated gain here named array gain. As a result, MU-MIMO approach has become as a complement segment for many modern communications standards, such as 802.11 (WiFi), 802.16 (WiMAX), LTE and LTE-A. The recent LTEAdvanced may have up to eight antenna pairs for the download link and up to four pairs for the upload link. For a long period of time, the problem of energy consumption essentially regarded autonomous, proceeded, or mobile communication terminals. 
During the last two decades, designing energy-saving communication terminals have become more important issue [3]. In addition to energy saving, energy-efficient systems have the advantage of mitigating the interference to other co-channel terminals as well as reducing surrounding effects such as heat dissipation and electronic pollution [4]. Therefore, current research works had focused on energy-efficiency (EE) in wireless communication systems since it is considered as one of the basis for every new design of $5 \mathrm{G}$ mobile communication systems.

In the last few years, worth research results that have been get for evaluating the energy efficiency of massive MIMO systems. For the uplink approach, authors of [1] show that massive MIMO has a significant feasible in improving energy efficiency by considering the power scaling law, which the energy efficiency is identified as the sum rate divided by the radiated power. This work results show that for achieving the same sum rate as a single antenna system, when $M \rightarrow \infty$, the transmit power of massive MIMO system reduces with the law of $1 / M$ if the BS has perfect channel state information (CSI) while with the law of $1 / \sqrt{M}$ if the BS has estimated channels with pilot contamination. For downlink approach, authors of [5] show that the optimal transmit power of the base station requires to increase with $\mathrm{M}$ in order to maximize the energy efficiency for a massive MIMO systems through zero forcing beam-forming (ZF) for the given numbers of antennas and users [6]. Note that the previous works on energy efficiency optimization have concentrated on either uplink approach or downlink approach. In contrast, there are some problems that extensive optimization so that the total energy efficiency is maximized for given fractions of uplink and downlink transmissions [5-6].

In this work, we will consider the energy efficiency (EE) for downlink massive MU-MIMO system, then we will evaluate its performance for different achievable rates considering improved versions of famous linear precoding techniques such as MRT (maximum ration transmission), and ZF (zero forcing). Then we will take into account some of system parameters as well the power constraints, number of antennas and number of users. The energy efficiency (EE) is integrated into the objective function called transmission efficiency which is combining from the main system parameters of the massive MU-MIMO. This work will come with a new a new proposed utility function that it will be subjected to the above defined objective function in order to have a significant reduction in transmit power consumption and optimize the number of antennas for a given number of users. Finally we will prove the existence of optimal combination of the system parameters so that the energy efficiency is maximized.

The rest of this paper is organized as follows: In Section 2, a literature review presented and discussed as compared to our work. Section 3 submitted a system model for Massive downlink MU-MIMO with imperfect CSI. Section 4 described our improved linear precoding techniques that will be used through our work. Section 5 describes the analysis of the achievable rates for our improved precoding schemes that be used here. Section 6 gives the description for our proposed model for maximizing the energy efficiency based on our improved linear precoding schemes. Section 7 showed the simulation results for our work. Conclusions that will be drawn from our work, is described in Section 8.

\section{Literature review}

All combination techniques of the method linear pre-coding in addition the massive array elements useful properties can be permitted and even suitable to enhance performance network in energy-efficiency also as well spectral-efficiency terms. Such combination concept will then forcibly and greatly bring network interference down or reduce and therefore enhance performance of system consequently. This is why MU-MIMO with large Mantenna has shown to save transmission-power under perfect-and-imperfect CSI in the uplink scenario [7]. In addition, it is also investigated in [7] that every single-antenna user-terminal transmit-power in massive MIMO scales down so proportionally to BS number of M-antenna with CSI perfect or in the case of CSI imperfect it scales down to square root BS number of M-antenna with imperfect-CSI using simple linear-techniques, targeting to obtain same performance like single-input-single-ouput (SISO) corresponding system. This results higher EE which is relatively important for next wireless-generations in which excessive power-consumption that is a key growing concern [8-9]. Besides [10] has also inspected that it is possible to reduce the transmitpower as square root of number of BS M-antenna accompany with only minor loss in data-rate under imperfect-CSI.. Energy-efficiency should be maximized while increasing transmitter power with M-antenna in order for compensating the everincreasing circuit-power-consumption [11].

The authors [4] showed that the EE metric is maximized by the opposite strategy of actually 
increasing the power with M-antenna, where the previous beliefs were considered inefficient regarding to EE scheme. The paper has considered a realistic consumption power of network and also the large scale of fading consideration. This realistic approach of EE optimization shows how the parameters interact such as number of M-antenna, active users also transmit-power including circuit power.

In addition, it has studied by [12] the beamforming training for CSI acquisition at each user side in massive downlink MU-MIMO, where it is found that the overhead for channel-estimation so can be small and also independent to BS number of Mantenna but proportional to number $\mathrm{K}$ terminals. It also derived achievable-rate for downlink using linear pre-coding schemes and compared the SE beam-forming training to [13] for no beamforming training. In [13] comparison between the conjugate and zero-forcing beam-forming in terms of $\mathrm{EE}$ and $\mathrm{SE}$ is done, where it is shown that for high $\mathrm{EE}$ conjugate-beam-forming is better while its SE is low, whereas the reverse holds for low-EE and high-SE for ZF. However, this paper considered only the radiated EE to evaluate network performance. In our model we will consider power-consumption of the analogcircuits power of the system.

The work of [14] shows the effect of vector and matrix normalizations on performance of system based on pre-coding normalization and under perfectCSI. In which the different pre-coding schemes were conducted to derive lower-and-upper-bound rates, where matched filter (MF) works well for matrix normalization while ZF performs better for vector normalization system, analysis and also comparison further are discussed. However, MF was further pointed out to perform well in de-centralized structure. While the work of [15] conducted by assuming imperfect-CSI with vector normalization consideration for analysis and comparison purpose.

For our model, we provide a novel consumptionpower and evaluate massive MIMO EE relying on our proposed model of consumption-power in a single-cellular network. After taking account circuitconsumption-power for transmission-antenna, the EE would not expect to be fundamentally increased gradually along with increase of BS M-antenna. For this vision, our model is further based on LTE Basestation transmission-power case with massive arrayelements. The loss factors and scale factor are applied such as DC-loss, cooling-loss, feeder-loss, main power-loss in our considered model [5].

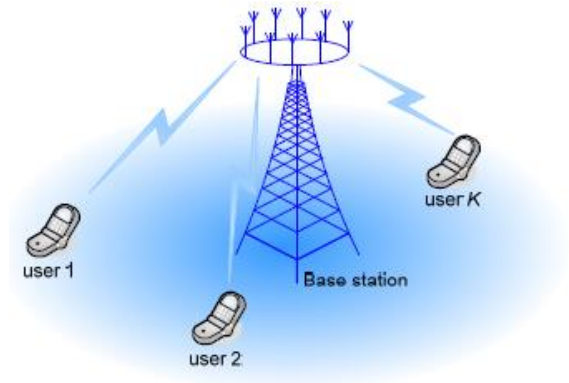

Figure. 1 Massive MU-MIMO downlink system model

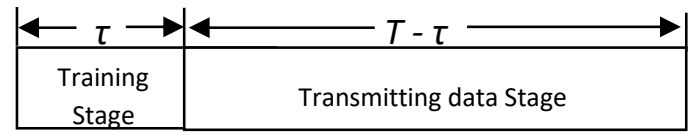

Figure. 2 Downlink transmission protocol

\section{System model}

We suppose an MU-MIMO downlink system as shown in Fig. 1 below, which contains just one base station served by $M$ antennas with $K$ single antenna users supposing that $\mathrm{K}$ users share the same timefrequency resources. The considered system has the massive property where $M \gg K$. We also suppose the channels staying unchanged within the coherent period $\tau$ of $T$ symbols. The transmitted procedure usually proceeds into double stages: training stage and transmitting data stage as shown in Fig. 2 below, note that $\tau$ interval is spent through training stage. At the training stage, BS predicts CSI by K-users benefiting from the downloaded signals through the uplink transmission. After that the BS deploys the received CSI with the provided linear precoding schemes to deal with the transmitting data.

Usually, the channel estimation process happens at downlink transmission, so that each terminal can estimate its operating channel by dealing with the pilot sequences that are transmitted from BS. Then, these terminals can feedback its CSI to its affiliated BS. However, channel estimation processes can overhead the BS working and this overhead is directly related to the number of antennas at this BS. As consequent, the massive MU-MIMO system's performance is degraded and over fatigued. Therefore, our work proposes that the channel is estimated at BS by uplink pilots, supposing channel reciprocity. Now, we consider the channel estimation is happened at $\tau$ duration, so each terminal has $\tau$ symbols of orthogonal pilot sequence and $\tau \geq K$. We can define the received pilot matrix $(M \times \tau)$ at $\mathrm{BS}$ and this matrix is given by:

$$
Y_{p}=\sqrt{P_{p}} H \Psi^{T}+N_{p}
$$


where $\Psi \epsilon(\tau \times K)$ is the pilot sequence deployed by $K$-users achieving $\Psi^{H} \Psi=I_{K}$ and $P_{p}=\tau P_{u}$, where $P_{u}$ is the transmitting power by any user. Using expression of (1), the channel can be estimated from as:

$$
\widehat{Y}_{p}=\sqrt{P_{p}} H+W
$$

where $\hat{Y}_{p}=Y_{p} \Psi^{*}$ and $W=N_{p} \Psi^{*}$. Let $y_{k}$ and $w_{k}$ be the $k^{\text {th }}$ columns of $\hat{Y}_{p}$ and $\mathrm{W}$ respectively, then:

$$
\hat{y}_{p, k}=\sqrt{P_{p}} h_{k}+w_{k}
$$

Then, we can get the final expression for channel estimation of $h_{k}$ as:

$$
\hat{h}_{k}=\frac{\sqrt{P_{p}}}{P_{p}+1} \hat{y}_{p, k}=\frac{P_{p}}{P_{p}+1} h_{k}+\frac{\sqrt{P_{p}}}{P_{p}+1} w_{k}
$$

With a big value of $P_{p}$ or $\tau$, it may have a perfect estimated CSI which it is the most important principle issue that it is considered in our work in this paper. So the base station considers the estimated CSI that are obtained from channel estimation process to handle the signals before sending them to $K$ users. Furthermore, linear precoding schemes are used and examined with our assumption that the BS deploys an imperfect CSI where this supposition is feasible and realistic for some cases such as high training power or high coherent interval $\tau$ which means consumes high $\tau$ for training stage.

Let $O \in \mathbb{C}^{M \times K}$ be a linear precoding matrix, and $x$ is a $K \times 1$ information vector, where $x_{k}$ is data symbol for $k^{\text {th }}$ user and $\mathbb{E}\left[\left|x_{k}\right|^{2}\right]=1$.The transmitted vector $s$ can be written as $s=O x$, and its average transmission power is constrained by $\mathbb{E}\left[\|s\|^{2}\right]=$ $\operatorname{tr}\left(O^{H} \mathrm{O}\right)=P_{t r}$. Then, the received vector at the $K$ is given by:

$$
y=H^{T} s+n
$$

where $n$ is a $K \times 1$ additive noise vector, where $n_{k} \in C \mathcal{N}(0,1)$. In our work, we adopt above model to obtain the linear precoding matrix in order to evaluate the performance of massive MU-MIMO system. The proposed system model is shown in Fig. 3 below.

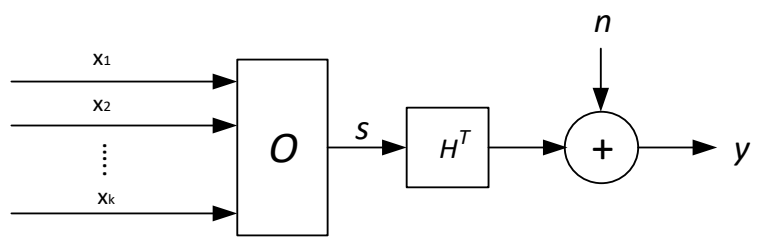

Figure. 3 Proposed system model

\section{Improved linear precoding schemes}

To enhance and evaluate the energy efficiency performance in massive MU-MIMO assuming imperfection base station BS channels state information. We supposed to consider two main and known linear precoding schemes called MRT (maximal-ratio-transmission) and ZF (zero-forcing), so we can do our enhancements and improvement to these two schemes taking into account power circuit consumption and transmit power considerations.

\subsection{Improved ZF precoding scheme}

In this work, we have done some contributions to Zero Forcing (ZF) precoding technique which it is an important scheme of linear precoding that it can cancel out the inter-user interference at each user through one cell. This precoding is supposed to execute a pseudo-inverse of the channel matrix $(H)$. $O_{Z F}$ can be given as:

$$
\begin{aligned}
O_{Z F} & =\frac{1}{\delta} H^{*}\left(H^{T} H^{*}\right)^{-1} \\
\delta & =\sqrt{\frac{\operatorname{tr}\left(v v^{H}\right)}{P_{t r}}} \\
v & =H^{*}\left(H^{T} H^{*}\right)^{-1}
\end{aligned}
$$

\subsection{Improved MRT precoding scheme}

Another one of the popular methods for increasing the signal-to-noise ratio (SNR) is considered in our work which it is a maximum-ratiotransmission (MRT) pre-coding scheme. We have also done some contributions to MRT precoding so as it can work very well in massive MU-MIMO system as the base station radiates low signal power to the users. $O_{M R T}$ can be given as:

$$
\begin{gathered}
O_{M R T}=\frac{1}{\delta} H^{*} \\
\delta=\sqrt{\frac{\operatorname{tr}\left(v v^{H}\right)}{P_{t r}}} \\
v=H^{*}
\end{gathered}
$$

\section{Achievable rate analysis}

To evaluate energy efficiency network performance, then we need to know achievable rate which follows the famous Shannon capacity as reference. It describes how maximum rate that can transmit through channel. Ergodic Channel is basically assumed and all the parameters are Gaussian random. In massive MIMO scheme 
ergodic $R_{\text {sum }}$ (sum rate) be chosen to describe its effectiveness. Due to simplicity analysis reason of spectral efficiency network we consider singlecellular where all user terminals share a constant transmit power equally in the downlink system. The ergodic achievable rate of $k^{\text {th }}$ user is then given as:

$$
R_{k}=E\left[\log _{2}\left(1+S I N R_{k}\right)\right](b i t s / s / H z)
$$

In this study, both sum-rate determination of improved pre-coders MRT and ZF are based on asymptotic convergence derivation, where their limiting SINR and SNR expressions respectively given by results provided with equal-power consideration scenarios.

\subsection{Achievable rate with ZF precoding}

From (5) and (6), we obtain the received vector with $\mathrm{ZF}$ as follows:

$$
\begin{gathered}
y=\frac{1}{\delta} H^{T} H^{*}\left(H^{T} H^{*}\right)^{-1} x+n \\
y=\frac{1}{\delta} x+n \\
H^{T} O_{Z F}=I_{K}
\end{gathered}
$$

Now, for $\mathrm{k}^{\text {th }}$ user, we have:

$$
\begin{gathered}
y_{k}=\frac{1}{\delta} x_{k}+n_{k} \\
\delta=\sqrt{\frac{\operatorname{tr}\left(H^{T} H^{*}\left(H^{T} H^{*}\right)^{-2}\right)}{P_{t r}}}=\sqrt{\frac{\operatorname{tr}\left(H^{T} H^{*}\right)^{-1}}{P_{t r}}}
\end{gathered}
$$

Then, the achievable rate of $k^{\text {th }}$ user with ZF is given by:

$$
R_{k}^{Z F}=\mathbb{E}\left[\log _{2}\left(1+\frac{\left|\frac{1}{\delta} x_{k}\right|^{2}}{\left|n_{k}\right|^{2}}\right)\right]
$$

\subsection{Achievable rate with MRT precoding}

From (5) and (9), the received vector with MRT can be expressed as:

$$
\begin{gathered}
y=\frac{1}{\delta} H^{T} H^{*} x+n \\
\delta=\sqrt{\frac{\operatorname{tr}\left(H^{T} H^{*}\right)}{P_{t r}}}
\end{gathered}
$$

Let $y_{k}, x_{k}$ and $n_{k}$ be the $k^{t h}$ elements of the $K$ $\times 1$ vectors $y, x$ and $n$ respectively and we define the $k^{\text {th }}$ column of $O_{M R T}$ as:

$$
O_{k}=h_{k}^{*}
$$

From (19), the received vector of $k^{\text {th }}$ user with MRT is given by:

$y_{k}=\frac{1}{\delta} h_{k}^{T} h_{k}^{*} x_{k}+\frac{1}{\delta} \sum_{i=1, i \neq k}^{K} h_{k}^{T} h_{i}^{*} x_{i}+n_{k}$

Then, the achievable rate of $k^{\text {th }}$ user with MRT is given by:

$$
R_{k}^{M R T}=\mathbb{E}\left[\log _{2}\left(1+\frac{\frac{1}{\delta^{2}}\left\|h_{k}\right\|^{4}}{1+\frac{1}{\delta^{2}} \sum_{i=1, i \neq k}^{K}\left|h_{k}^{T} h_{i}^{*}\right|^{2}}\right)\right]
$$

\section{Energy efficiency analysis}

During the last two decades, designing energysaving communication terminals have become a very important issue. In addition to energy saving, energyefficient systems have the advantage of mitigating the interference to other co-channel terminals as well as reducing surrounding effects such as heat dissipation and electronic pollution. Therefore, recent research works had focused on energy-efficiency (EE) in wireless communication systems since the energy efficiency (EE) is considered as one of the critical design goals for $5 \mathrm{G}$ networks.

To offer the exact required data rate for a terminal or the required sum rate for a whole system, massive MIMO systems are anticipated to achieve these requirement, since the transmitted power can be remarkably downgraded due to the large array gain and multiplexing gain. The downlink energy efficiency is determined by dividing the total cell throughput for $\mathrm{i}^{\text {th }}$-cell to the total power consumption of this cell, where the average achievable or through is the achievable sum rate of all users $K$ exempting the uplink training overhead. So the downlink energy efficiency (EE) can be stated as:

$$
E E=\frac{\sum_{i=1, i \neq k}^{K} R_{k}}{\frac{P_{t r}}{2 \rho_{R F}}\left(1-\frac{\tau}{T}\right)+\frac{2 f}{\rho_{C}}+M P_{N}}
$$

where $\rho_{R F}$ is the RF power amplifier efficiency, while $\rho_{C}$ is the power efficiency of computing measured in flops $/ W$, and $P_{N}$ is the internal non-RF power consumption related to each antenna associating to analog electronic devices including $\mathrm{A} / \mathrm{D}$ and $\mathrm{D} / \mathrm{A}$ converters. $f$ is the floating-point operations persecond (flops) per-antenna for each user, which it can be considered as the total computation complexity for channel estimation and precoding technique: it becomes $710 G_{\text {flops }}$ with ZF and 239 Gflops with MRT 
[5]. These values are all within a realistic scope and the enormous majority of the computations can be parallelized for each antenna.

For simplicity, we can rewrite expression (24) as:

$$
E E=\frac{\sum_{i=1, i \neq k}^{K} R_{k}}{A P_{t r}+B+C M}
$$

where $A=\frac{\left(1-\frac{\tau}{T}\right)}{2 \rho_{R F}}, B=\frac{2 f}{\rho_{c}}$ and $C=P_{N}$. Now, let us get the final expressions for energy efficiencies with $\mathrm{ZF}$ and MRT respectively.

\subsection{Energy efficiency with ZF}

The achievable sum rate for $\mathrm{ZF}$ can be derived as follows:

$$
\begin{aligned}
R^{Z F}=\sum_{k=1}^{K} R_{k}^{Z F} & R^{Z F}=\sum_{k=1}^{K} \mathbb{E}\left[\log _{2}\left(1+\frac{\left|\frac{1}{\delta} x\right|^{2}}{\left|n_{k}\right|^{2}}\right)\right] \\
& =K \log _{2}\left(1+\mathbb{E}\left[\frac{\left|\frac{1}{\delta} x\right|^{2}}{\left|n_{k}\right|^{2}}\right]\right) \\
R^{Z F} & =K \log _{2}\left(1+\mathbb{E}\left[\frac{P_{t r}}{\operatorname{tr}\left(H^{T} H^{*}\right)^{-1}}\right]\right)
\end{aligned}
$$

Using Lemma 2 from definition (See Appendix), then we obtain:

$$
R^{Z F}=K \log _{2}\left(1+\frac{P_{t r}}{\frac{K}{M-K}}\right)
$$

Then, we have the energy efficiency with ZF as:

$$
E E_{Z F}=\frac{R^{Z F}}{P_{t r} A+B+M C}
$$

\subsection{Energy efficiency with MRT}

The achievable sum rate for MRT can be derived as follows:

$$
R^{M R T}=\sum_{k=1}^{K} R_{k}^{M R T}
$$

Using (23), the above expression will be given as:

$$
R^{M R T}=\sum_{k=1}^{K} \mathbb{E}\left[\log _{2}\left(1+\frac{\frac{1}{\delta^{2}}\left\|h_{k}\right\|^{4}}{1+\frac{1}{\delta^{2}} \sum_{i=1, i \neq k}^{K}\left|h_{k}^{T} h_{i}^{*}\right|^{2}}\right)\right]
$$

$$
R^{M R T}=K \log _{2}\left(1+\left(\mathbb{E}\left[\frac{1+\frac{1}{\delta^{2}} \sum_{i=1, i \neq k}^{K}\left|h_{k}^{T} h_{i}^{*}\right|^{2}}{\frac{1}{\delta^{2}}\left\|h_{k}\right\|^{4}}\right]\right)^{-1}\right)
$$

where $=\sqrt{\frac{\operatorname{tr}\left(H^{T} H^{*}\right)}{P_{t r}}}$, then, $\delta^{2}=\frac{\operatorname{tr}\left(H^{T} H^{*}\right)}{P_{t r}}$ and $\frac{1}{\delta^{2}}=$ $P_{t r} \operatorname{tr}\left(H^{T} H^{*}\right)^{-1}$. Then (33) becomes:

$$
\begin{aligned}
& R^{M R T}= \\
& K \log _{2}\left(1+\left(E\left[\frac{1+P_{t r} \operatorname{tr}\left(H^{T} H^{*}\right)^{-1} \sum_{i=1, i \neq k}^{K}\left|h_{k}^{T} h_{i}^{*}\right|^{2}}{P_{t r} \operatorname{tr}\left(H^{T} H^{*}\right)^{-1}\left\|h_{k}\right\|^{4}}\right]\right)^{-1}\right)
\end{aligned}
$$

$$
\begin{aligned}
& R^{M R T}= \\
& K \log _{2}\left(1+\left(E\left[\frac{1+P_{t r} \operatorname{tr}\left(H^{T} H^{*}\right)^{-1} \sum_{i=1, i \neq k}^{K} \frac{\left|h_{k}^{T} h_{i}^{*}\right|^{2}}{\left\|h_{k}\right\|^{2}}}{P_{t r} \operatorname{tr}\left(H^{T} H^{*}\right)^{-1}\left\|h_{k}\right\|^{2}}\right]\right)^{-1}\right)
\end{aligned}
$$

Let $\tilde{h}_{i}=\frac{\left|h_{k}^{T} h_{i}^{*}\right|}{\left\|h_{k}\right\|}$, where $\tilde{h}_{i}$ is a Gaussian random variable with zero-mean and unity variance and does not depend on $h_{k}$. So, we have:

$$
\begin{gathered}
R^{M R T}= \\
K \log _{2}\left(1+\left(\mathbb{E}\left[\frac{\frac{1}{\left\|h_{k}\right\|^{2}+P \operatorname{trt} t\left(H^{T} H^{*}\right)^{-1} \sum_{i=1, i \neq k}^{K}\left|\widetilde{h}_{i}\right|^{2}}}{P \operatorname{trtr}\left(H^{T} H^{*}\right)^{-1}\left\|h_{k}\right\|^{2}}\right]\right)^{-1}\right)
\end{gathered}
$$

To simplify the numerator of (36), we will use the definition and Lemma 1 (See Appendix) for first part of numerator, and then we have:

$$
\mathbb{E}\left[\frac{1}{\left\|h_{k}\right\|^{2}}\right]=\frac{1}{M(M+1)}
$$

To simplify the second part of the numerator, we will use Lemma 2 from definition (See Appendix), as a result:

$$
\begin{aligned}
& \mathbb{E}\left[\frac{1}{\left\|h_{k}\right\|^{2}}+P_{t r} \operatorname{tr}\left(H^{T} H^{*}\right)^{-1} \sum_{i=1, i \neq k}^{K}\left|\tilde{h}_{i}\right|^{2}\right] \\
= & \frac{1}{M(M+1)}+P_{t r} \mathbb{E}\left[\operatorname{tr}\left(H^{T} H^{*}\right)^{-1} \sum_{i=1, i \neq k}^{K}\left|\tilde{h}_{i}\right|^{2}\right] \\
= & \frac{1}{M(M+1)}+P_{t r}\left(\frac{K}{M-K}\right)
\end{aligned}
$$

Now, to simplify the denominator of (35), we will also use Lemmas 1 and 2 from definition (See Appendix), with $M$ degree of freedom $(M>K)$, we obtain: 


$$
\mathbb{E}\left[\frac{1}{P_{t r} \operatorname{tr}\left(H^{T} H^{*}\right)^{-1}\left\|h_{k}\right\|^{2}}\right]=\frac{1}{P_{t r}\left(\frac{K}{M-K}\right) M(M+1)}
$$

Then, the final expression for $R^{M R T}$ is given by:

$$
R^{M R T}=K \log _{2}\left(1+\frac{P_{t r}\left(\frac{K}{M-K}\right) M(M+1)}{\frac{1}{M(M+1)}+P_{t r}\left(\frac{K}{M-K}\right)}\right)
$$

So, the energy efficiency with MRT can be expressed as:

$$
E E_{M R T}=\frac{R^{M R T}}{P_{t r} A+B+M C}
$$

\section{Simulation results}

The parameters used for performance evaluation of our proposed model for energy-efficiency with improved linear precoding schemes are shown in Table 1 below. Besides, those parameters highlighted in table, their values are taken by referring or according to [5]. Since the authors concerned the circuit consumption-power and the relative loss factors values which will be then used to evaluate energy-efficiency precoding performance. In all the scenarios, noise power at all receivers' side is assumed to be one.

To evaluate precoding performance of our propose model, we provide results based on simulation of energy-efficiency and also spectralefficiency as well, and compare different precoders performance and also differ to beliefs that circuit power is a fixed power which it is clear noted at [7] and [12]. To illustrate behavior of EE in contrast to where circuit-power consideration was ignored with very-large BS M-antenna energy-consumption model design in the literatures such as [4] and [11].

The Figs. 4 and 5 illustrate the energy-efficiency versus BS number of M-antenna where the system serves 15 user terminals simultaneously with CSI imperfect, assuming $\left(\xi^{2}=1\right)$ channel error estimation and $10 \mathrm{~dB}$ transmit power, other parameters are found by Table 1 .

In this proposed model, the energy-efficiency precoding performance for the two different linear precoding, that are, MRT and ZF, are compared. The proposed model evaluates energy-efficiency based on circuit consumption power concept and very-large $\mathrm{BS}$ number of M-antenna. Hence, results show as BS number of $\mathrm{M}$-antenna increases then both precoding performances increase at first. Realistic EE model behaves firstly appearing to increase until their maximum values by increasing $\mathrm{M}$-antenna and then it decreases later due to growing of circuit consumption power which grows linearly so with $\mathrm{M}$ -
Table 1. System parameters

\begin{tabular}{|c|c|c|}
\hline Description & $\begin{array}{c}\text { Symb } \\
\text { ol }\end{array}$ & $\begin{array}{c}\text { Value } \\
(\mathbf{m W})\end{array}$ \\
\hline Consumed power in DAC convertor & $P_{D A C}$ & 15.6 \\
\hline Consumed power in mixer & $P_{M I X}$ & 30.3 \\
\hline Consumed power in filter at Tx & $P_{F I L T}$ & 20 \\
\hline Consumed power in LO & $P_{S Y N}$ & 50 \\
\hline Loss due to feeder of antenna & $\sigma_{\text {feed }}$ & 0.5 \\
\hline Loss due to direct-current power & $\sigma_{D C}$ & 0.06 \\
\hline Loss due to main supply power & $\sigma_{M S}$ & 0.07 \\
\hline Loss due to system cooling & $\sigma_{C o o l}$ & 0.09 \\
\hline Efficiency of PA & $\eta$ & 0.38 \\
\hline
\end{tabular}

antenna as shown in Fig. 5.

In Fig. 4 accordingly, when BS has small number of M-antenna ( $\operatorname{cmall} \alpha=M / K$ ) compared to number of $K$ user terminals then MRT precoding performance is better than $\mathrm{ZF}$ pre-coding performance in that particular scenario belonging to low-SNR region, although the gap is slightly modest. While the ZF precoding performance outperforms MRT precoder one in the scenario of large BS number of M-antenna (large $\alpha=M / K$ ) and the gap is slightly important as Mantenna increases.

Fig. 5 illustrates that although energy-efficiency precoding performance of both precoders increase but later on decrease due to increase of the circuit consumption power that grows linearly so with Mantenna. From observation we then see that even though increasing spectral-efficiency may not able to compensate additional consumption power. Our results further confirm that the energy-efficiency is bounded instead of unbounded contrasting certain thinking observe in the literature (circuitpower=fixed-power) in which energy-efficiency increases without bound because they assume that circuit power is a fixed power even with increasing BS number of M-antenna, we can see that such assumption does not really hold. In other word, existence of M-antenna cross-point is driven to take the decision about each pre-coding performance achievement as shown in most of the figures that if the active BS number of $\mathrm{M}$-antenna is larger compared to the M-antenna cross point ( $\left.M_{C R O S S}\right)$ then the $\mathrm{ZF}$ precoder reaches higher $\mathrm{EE}$, however, in turn if the number of active BS M-antenna is smaller compared to M-antenna cross-point ( $\left.M_{C R O S S}\right)$ then the MRT precoder reaches higher EE than ZF pre-coding performance.

In Fig. 6, we plot EE of the two precoders where 15 user terminals are served simultaneously with imperfect CSI assuming $\left(\xi^{2}=0.50\right)$ channel error estimation and also $10 \mathrm{~dB}$ given transmit power. The results show MRT precoding performance 


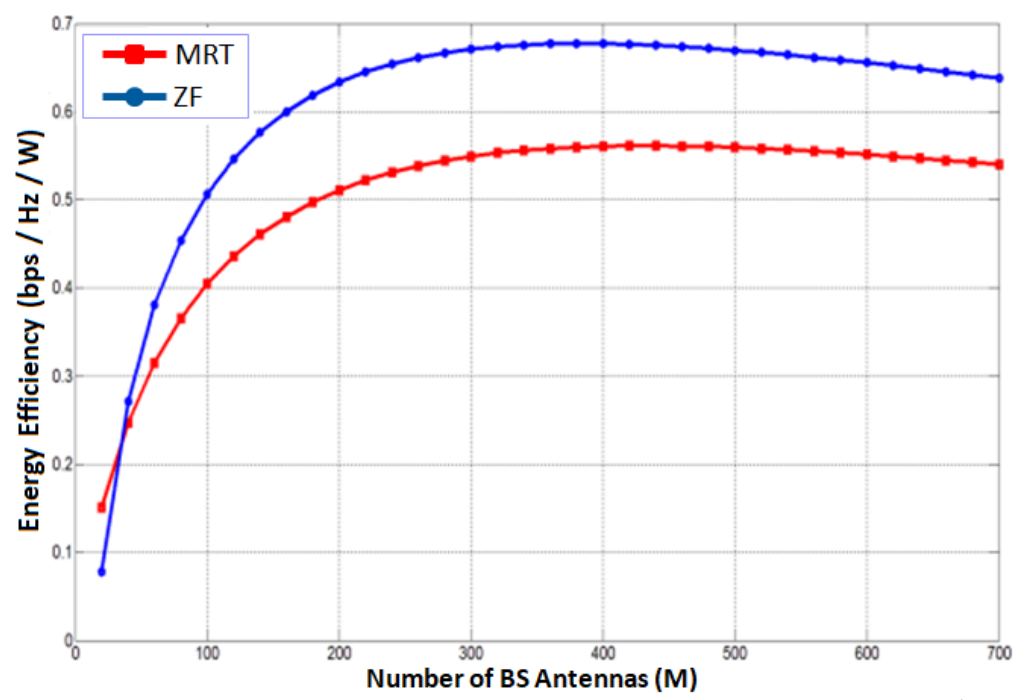

Figure. 4 Energy-efficiency precoding performance $\left(\rho_{\mathrm{d}}=10 \mathrm{~dB}, \mathrm{~K}=15, \xi^{2}=0.50\right)$

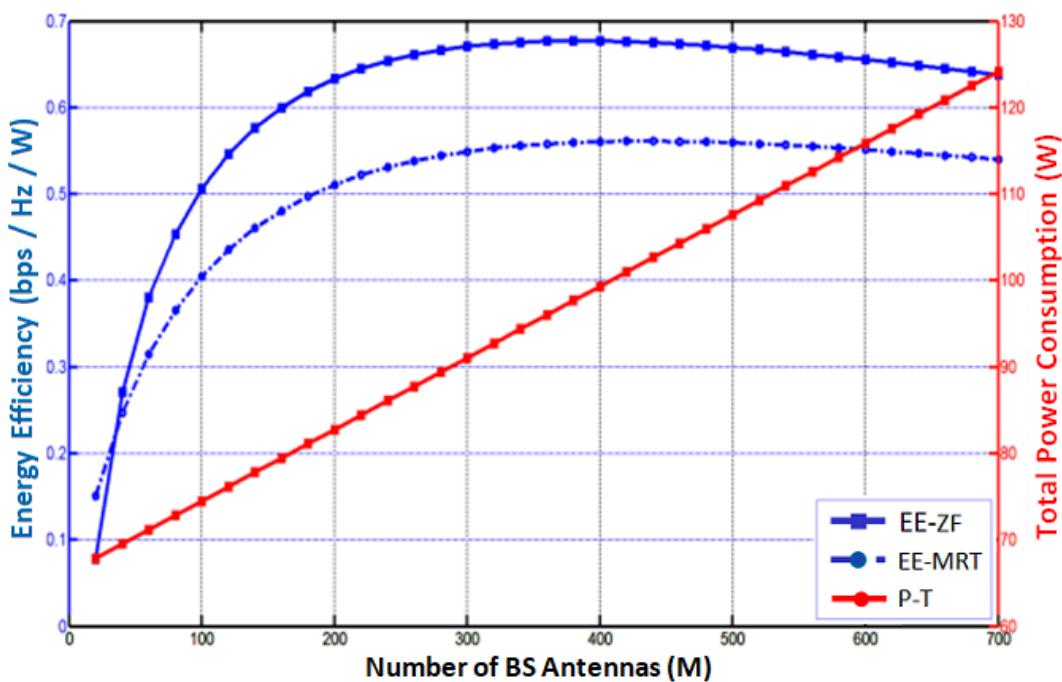

Figure. 5 Energy-efficiency precoding performance and total consumption power $\left(K=15, \xi^{2}=0.50, \rho_{d}=10 d B\right)$

outperforms ZF precoding performance within small BS number of M-antenna deployment comparatively to number of $K$ terminals (from 20 to approximately 40 antennas). Whereas ZF precoding performance is better than MRT one when BS number of M-antenna is very-large compared to number of $K$ terminals in which the gap between them is slightly considerable compared to MRT scheme one. In contrast to this belief in literature, our model shows that EE is not unbounded when M-antenna increases very-large. Furthermore, we observe the optimal EE value for each precoder, approximately with $0.6773 \mathrm{bps} / \mathrm{W} / \mathrm{Hz}$ using 380 antennas in service while $0.5612 \mathrm{bps} / \mathrm{W} / \mathrm{Hz}$ with 440 antennas, respectively for ZF and MRT precoders. Even though, our model of EE increases, but later on it decreases. Compared to scenario where the circuit consumption power is not taken account the EE increases infinitely as M-antenna increases, we prove further that this belief does not really hold true practically, and also validated by our proposed model.

In Fig. 7, we plot the energy-efficiency of two precoders versus BS number of M-antenna with two different number of user terminals $(K=10 \& 30)$ that are simultaneously served under $10 \mathrm{~dB}$ transmitpower with CSI imperfect assuming $\left(\xi^{2}=0.50\right)$ for channel estimation error. The results demonstrate and confirm that an efficient-energy can be achieved by allowing so more and more users to access the channel simultaneously, thanks to massive MIMO that illustrates one $5 \mathrm{G}$ example expectations. We observe the energy-efficiency performance that accommodates 30 users is slightly higher compared to 10 users' accommodation. Additionally, performance gap increases between two schemes as terminals number increased. Both cases, ZF 


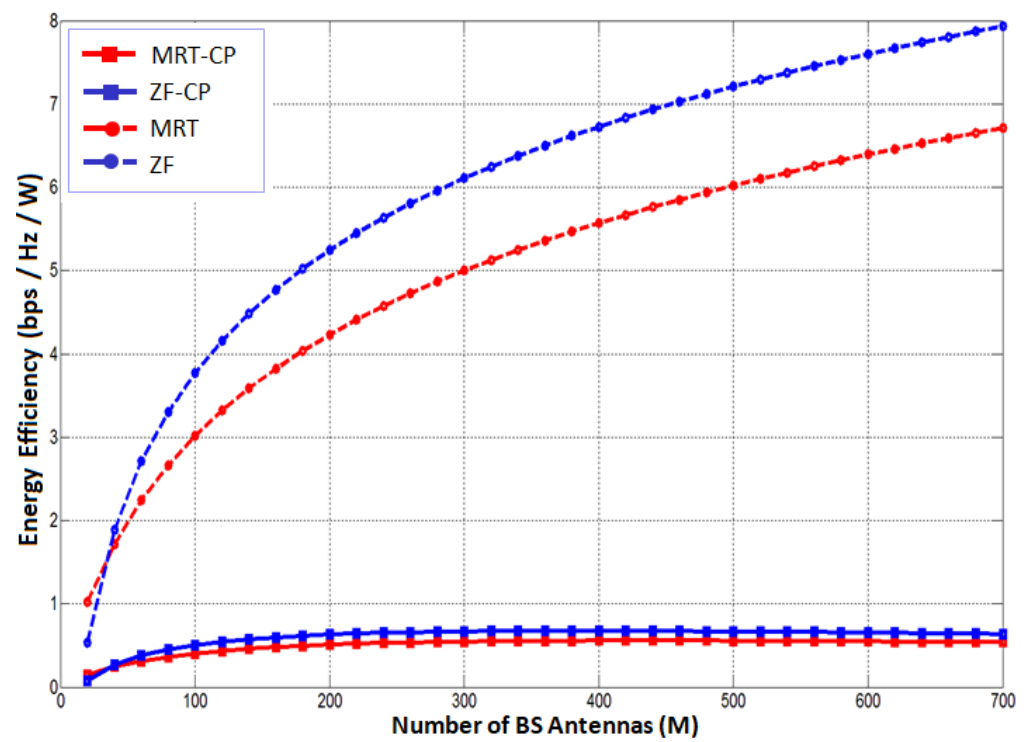

Figure. 6 Energy-efficiency precoding performance $\left(K=15, \xi^{2}=0.50, \rho_{d}=10 d B\right)$

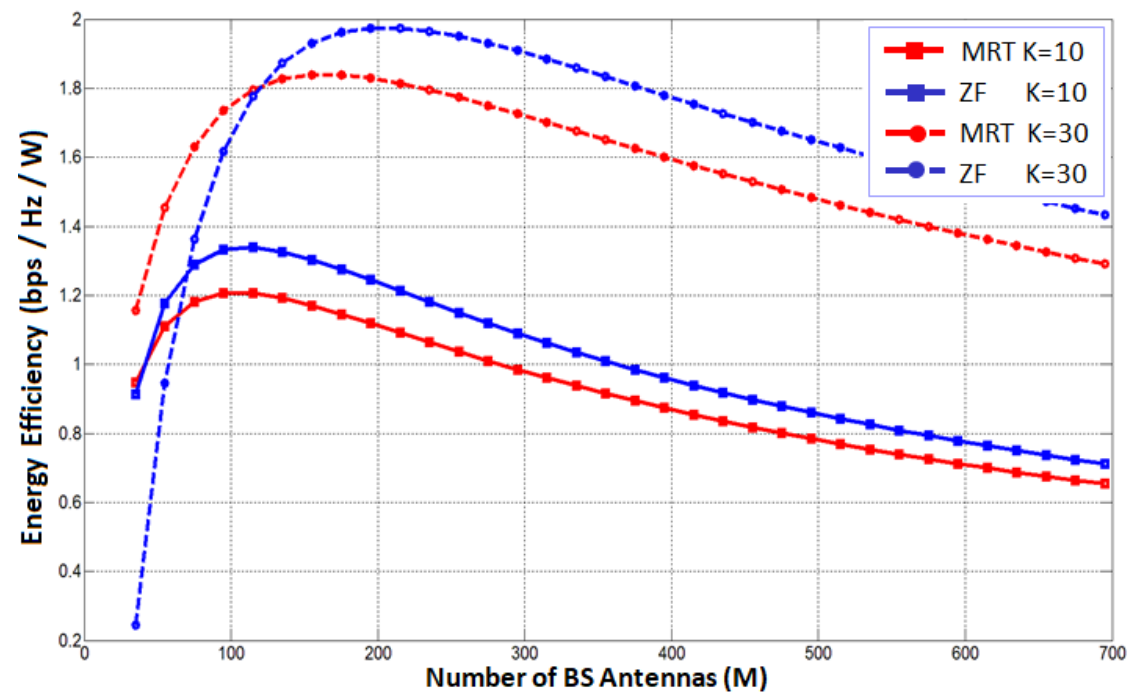

Figure. 7 Energy-efficiency comparison precoding performance $\left(\rho_{d}=0 d B, K=10 \& 30, \xi^{2}=0.50\right)$

precoding performance outperforms MRT within large number of service BS M-antenna comparatively to number of $K$ user terminals belonging to high-SNR region while MRT outperforms ZF within a small range number of service $\mathrm{BS} \mathrm{M}$-antenna belonging to low-SNR region, where M-antenna cross-point is used to compare the performance of precoding two schemes. Both cases, at first energy-efficiency performance increases when BS M-antenna service increases and then decreases later because of circuit consumption power increases linearly so with $M$ antennas as Fig. 7 shown.

In Fig. 7, we plot the energy-efficiency of two precoders versus BS number of $\mathrm{M}$-antenna with two different number of user terminals $(K=10 \& 30)$ that are simultaneously served under $10 \mathrm{~dB}$ transmitpower with CSI imperfect assuming $\left(\xi^{2}=0.50\right)$ for channel estimation error. The results demonstrate and confirm that an efficient-energy can be achieved by allowing so more and more users to access the channel simultaneously, thanks to massive MIMO that illustrates one $5 \mathrm{G}$ example expectations. We observe the energy-efficiency performance that accommodates 30 users is slightly higher compared to 10 users' accommodation. Add itionally, performance gap increases between two schemes as terminals number increased. Both cases, ZF precoding performance outperforms MRT within large number of service BS M-antenna comparatively to number of $K$ user terminals belonging to high-SNR region while MRT outperforms ZF within a small range number of service BS $\mathrm{M}$-antenna belonging to low-SNR region, where M-antenna cross-point is used to compare the performance of precoding two 
schemes. Both cases, at first energy-efficiency performance increases when BS M-antenna service increases and then decreases later because of circuit consumption power increases linearly so with $M$ antennas as Fig. 7 shown

Fig. 8 illustrates relationship between energyefficiency and number of $K$ terminals by fixing BS number of M-antenna to 150 and transmit power to $10 \mathrm{~dB}$ with CSI imperfect assuming $\left(\xi^{2}=0.50\right)$ for channel error estimation. The curves show that the energy-efficiency can increase at beginning by increasing $K$ number of terminals; we also observe an appropriate value so that energy-efficiency can let be maximized. Besides, the energy-efficiency of $\mathrm{ZF}$ precoding performance grows first and then decreases later. This reason explains fact that when $\mathrm{ZF}$ precoding is performed then it must exist one optimal $K$ number of terminals for the highest EE. Furthermore, another observation from the curves is that the ZF precoding performance falls down quickly under MRT precoding performance that was best efficiently before because of $\mathrm{K}$ number of user terminals is becoming increasingly closer to BS number of $\mathrm{M}$-antenna that will not fulfill the inequality assumption as required by $\mathrm{ZF}$, that is, BS M-antenna should be very large compared to $K$ user terminals, $M>>K$.

\section{Conclusions}

This work has covered precoding performance taking account the parameters as EE, total circuit consumption power and the radiated power, while also tradeoff phenomenon between SE-EE for MRT/ZF schemes had been considered. Simulation results showed that the improved $\mathrm{ZF}$ precoding scheme achieves higher EE as compared to improved MRT precoding scheme, while the results showed that improved MRT precoding outperforms ZF precoding within small BS number of $\mathrm{M}$-antenna deployment comparatively to number of $\mathrm{K}$ terminals (from 20 to approximately 40 antennas). The results also demonstrated and confirmed that an efficientenergy can be achieved by allowing so more and more users to access the channel simultaneously. Furthermore, results confirmed the emitted-power as antennas increase in opposite to the growing of circuit power. Besides, it showed that MRT requires less transmit power when system has low data-rate that is shared equally among all terminals, this makes MRT precoding to be best efficient power choice than $\mathrm{ZF}$ precoding. However, the ZF precoding is best choice of efficient power when inversely the system has high capacity that is shared equally among terminals.
As fifth generation network is expected widely to provide some varieties of services. This work was concentrated on energy-efficiency linear precoding performance evaluation of massive MIMO singlecellular systems. Therefore, the future scope will be focused on multi-cellular systems by considering both the reverse-link and forward-link to model/analyze linear precoding energy efficiency, which will take especially into account Pico-cellular BS or Femto-cellular BS deployment within Macrocellular BS in order so to satisfy the hotspot users and improve the network coverage/link reliability under efficient utilization power.

\section{Appendix - "Central Wishart Matrix [16]"}

The $n \times n$ random matrix which satisfies $A=$ $H H^{H}$ is a real/complex central Wishart matrix with $n$ degrees of freedom and covariance matrix $\mathfrak{S}$, $\left(A \sim W_{m}(n, \subseteq)\right)$, while the matrix $H$ has columns are zero-mean independent real/complex Gaussian vectors with covariance matrix $\mathfrak{S}$. This matrix is satisfying the following two lemmas:

\section{Lemma 1:}

For a Central Wishart Matrix $W \sim W_{m}(n, I)$ :

$\mathbb{E}[\operatorname{tr}\{W\}]=m n$

$\mathbb{E}\left[\operatorname{tr}\left\{W^{2}\right\}\right]=m n(m+n)$

$\mathbb{E}\left[\operatorname{tr}^{2}\{W\}\right]=m n(m n+1)$

\section{Lemma 2:}

For a Central Wishart Matrix $W \sim W_{m}(n, I)$, with $n>m$ :

$\mathbb{E}\left[\operatorname{tr}\left\{W^{-1}\right\}\right]=\frac{m}{n-m}$

\section{Conflicts of Interest}

The authors declare no conflict of interest.

\section{Author Contributions}

The paper conceptualization, methodology, analysis, investigation, simulation, discussion, writing-original draft preparation, writing-review and editing, have been done by one author (Dheyaa Jasim Kadhim).

\section{List of Notations}

$K \quad$ : Number of users

$M \quad$ : Number of antennas

$\tau \quad$ : Duration of cannel estimation 
$Y_{p} \quad$ : Received pilot matrix

$H \quad$ : Channel matrix

$\Psi \quad$ : Pilot sequence

$P_{u} \quad$ : Transmitted power per user

$N_{p} \quad$ : Noise Power

$P_{p} \quad:$ Received power per $\tau$

$\hat{Y}_{p} \quad$ : Estimated received pilot matrix

$\hat{h}_{k} \quad$ : Estimated channel matrix per k-user

$O \quad$ : Linear precoding matrix

$P_{t r} \quad$ : Average transmitted power

$R_{k} \quad$ : Ergodic achievable rate of k-user

$\rho_{R F} \quad$ : RF power amplifier efficiency

$\rho_{C} \quad:$ Measured power efficiency in flops/W

$P_{N} \quad$ : Internal non-RF power consumption

$\xi^{2} \quad$ : Channel estimation error

$\rho_{d} \quad$ : Transmitted power in $\mathrm{dB}$

\section{References}

[1] N. Prasad, H. Zhang, H. Zhu, and S. Rangarajan, "Multi-user MIMO scheduling in the fourth generation cellular uplink", IEEE Transaction on Wireless Communications, Vol. 12, No. 9, pp. 4272-4285, 2013.

[2] J. Zhang, S. Chen, Y. Lin, J. Zheng, B. Ai, and L. Hanzo, "Cell-free massive MIMO: A new nextgeneration paradigm", IEEE Access Journal, Vol. 7, pp. 99878-99888, 2019.

[3] H. Yang and T. L. Marzetta, "Energy efficiency of massive MIMO: Cell-free vs. cellular", In: Proc. of 87th IEEE Vehicular Technology Conf. (VTC Spring), Porto, Portugal, pp. 1-5, 2018.

[4] E. Bjornson, L. Sanguinetti, J. Hoydis, and M. Debbah, "Designing multi-user MIMO for energy efficiency: when is massive MIMO the answer?", In: Proc. of IEEE Wireless Communications and Networking Conf. (WCNC), Istanbul, Turkey, pp. 242-247, 2014.

[5] T. Parfait, Y. Kuang, and K. Jerry, "Performance analysis and comparison of ZF and MRT based downlink massive MIMO systems", In: Proc. of $6^{\text {th }}$ International Conf. on Ubiquitous and Future Networks (ICUFN), Shanghai, China, pp. 383388, 2014.

[6] P. Liu, K. Luo, D. Chen, and T. Jiang, "Spectral efficiency analysis of cell-free massive MIMO systems with zero-forcing detector", IEEE Transactions on Wireless Communications, Vol. 19, No. 2, pp. 795-807, 2019.

[7] H. Q. Ngo, E. G. Larsson, and T. L. Marzetta, "Energy and spectral efficiency of very large multiuser MIMO systems", IEEE Transactions on Communications, Vol. 61, No. 4, pp. 14361449, 2013.
[8] R. A. Aljiznawi, N. H. Alkhazaali, S. Q. Jabbar, and D. J. Kadhim, "Quality of service (qos) for 5G networks", International Journal of Future Computer and Communication, Vol. 6, No. 1, p. 27, 2017.

[9] W. A. Mahmoud and D. J. Kadhim. "A Proposal Algorithm to Solve Delay Constraint Least Cost Optimization Problem", Journal of Engineering, Vol. 19, No. 1, pp. 155-160, 2013.

[10] T. X. Tran and K.C. Teh, "Spectral and energy efficiency analysis for SLNR precoding in massive MIMO systems with imperfect CSI", IEEE Transactions on Wireless Communications, Vol. 17, No. 6, pp. 4017-4027, 2018.

[11] T. A. Khan, A. Yazdan, and R. W. Heath, "Optimization of power transfer efficiency and energy efficiency for wireless-powered systems with massive MIMO", IEEE Transactions on Wireless Communications, Vol. 17, No. 11, pp. 7159-7172, 2018.

[12] H. Q. Ngo, E. G. Larsson, and T. L. Marzetta, "Massive MU-MIMO downlink TDD systems with linear precoding and downlink pilots", In: Proc. of 51st Annual Allerton Conf. on Communication, Control, and Computing, Allerton, Monticello, USA, pp. 293-298, 2013.

[13] H. Yang and T. L. Marzetta, "Performance of conjugate and zero-forcing beamforming in large-scale antenna systems", IEEE Journal on Selected Areas in Communications, Vol. 31, No. 2, pp. 172-179, 2013.

[14] Y. Liu, J. Liu, Q. Wu, Y. Zhang, and M. Jin, “A near-optimal iterative linear precoding with low complexity for massive MIMO systems", IEEE Communications Letters, Vol. 23, No. 6, pp. 1105-1108, 2019.

[15] A. A. Abdulateef, S. M. Ibrahim, A. H. Mohammed, and I. A. Abdulateef, "Performance Analyses of Channel Estimation and Precoding for Massive MIMO Downlink in the TDD System", In: $4^{\text {th }}$ International Symposium on Multidisciplinary Studies and Innovative Technologies (ISMSIT), Istanbul, Turkey, pp. 1-6, 2020.

[16] A. N. Bishop, P. Del Moral, and A. Niclas, "An introduction to Wishart matrix moments", arXiv preprint arXiv:1710.10864. 